\title{
TeV-PeV neutrinos from accretion disks around super-massive black holes in active galaxies
}

\author{
Wlodek Bednarek \\ Department of Astrophysics, University of Lodz, Lodz, Poland \\ E-mail: bednar@uni.lodz.pl
}

\begin{abstract}
We propose that TeV-PeV neutrinos, recently detected by the IceCube, can be produced by neutrons interacting with the matter of the accretion disks around black holes in active galaxies. Neutrons are extracted from nuclei which are disintegrated in jets of active galaxies. The neutrino spectra, expected from the whole population of the active galaxies, are confronted with the observations. We propose that neutrinos produced in such scenario can explain the extra-galactic neutrino background recently measured by the IceCube neutrino detector, provided that $5 \%$ fraction of galaxies is AGN and a few percent of neutrons reach the accretion disk.
\end{abstract}

35th International Cosmic Ray Conference - ICRC2017-

10-20 July, 2017

Bexco, Busan, Korea 


\section{Introduction}

Recently a few tens of neutrino events have been detected by the IceCube neutrino telescope in the TeV-PeV energy range [1], 2]. It has been proposed that these neutrinos are produced within active galaxies at cosmological distances (see for review [3, 那. We follow the general scenario, proposed in [5], that protons accelerated in the jet interact with the accretion disk radiation. However, we consider more complicated scenario in which neutrinos are produced by neutrons colliding with the accretion disk matter. We note that a significant part of the matter which accretes onto the super-massive black hole (SMHB) in the AGN nucleus has to be composed from nuclei. This matter is likely to be more abundant in heavy nuclei, in respect to primordial matter containing $~ 25 \%$ of helium, due to the efficient star formation occurring in the central parts of the parent galaxies. These nuclei are expected to be accelerated to relativistic energies in the re-connection regions in the jet and/or at the jet boundary layer. The nuclei disintegrate in collisions with the accretion disk radiation producing relativistic neutrons which, since neutral, can easily find the way to the dense matter in the accretion disk. Note that the neutrons from the disintegration of the nuclei have energies more than an order of magnitude lower than the neutrons produced in collisions of protons with photons due to the lower threshold on the first process. Therefore, neutrons from nuclei can easily produce multi- $\mathrm{TeV}$ neutrinos in consistency with the IceCube observations (see details in [6]).

\section{Nuclei in the jet}

We consider the processes in the inner part of the active galaxy in which jets are expected to be launched by a rotating black hole or from the inner part of the accretion disk [7, 8, 9]. In fact, both processes mentioned above can play an important role. Therefore, the jet can be composed of a faster moving core region and slowly moving sheath or a cocoon formed by the matter surrounding the jet or by the outer layers of the jet expelled from the accretion disk, i.e. the accretion disk wind. We consider two regions as responsible for the acceleration of particles in the jet/cocoon system, i.e. the re-connection regions in the jet and the transition region between the jet and the cocoon. We assume that an essential part of the jet power can be transferred to the relativistic nuclei in the inner part of the jet. As a result of the disintegration of nuclei in collisions with the disk radiation, relativistic neutrons are extracted. In fact, neutrons can take a significant part of the energy of the accelerated nuclei, between $\sim 8^{-1}$ (in the case of the presence of only primordial Helium, assuming the primordial He abundance of $\sim 0.25$ [10], and up to $\sim 2^{-1}$ (if accelerated hadrons are dominated by heavy nuclei).

We assume that nuclei can be accelerated in re-connection regions, which are oriented in the general direction towards the accretion disk in the SMBH rest frame. Their energies are large enough that the disintegration process is efficient. Acceleration of particles in the re-connection regions is expected to be responsible for the acceleration of particles in relativistic jets of blazars 111, 12, 13, 14, 15, 16, 17]. We apply a simple model for the acceleration process in the reconnection region. Its length, $L_{\mathrm{rec}}$, scales with the distance from the base of the jet [18]. The distance along the jet, $R$, is given in units of the inner radius of the jet, $R_{\text {in }}$, according to $r=$ $R / R_{\text {in }}$. Then, $L_{\text {rec }}$ is linked to the SMBH mass, $L_{\text {rec }}=\xi R_{\text {in }} r \approx 10^{14} \xi_{-1} M_{9} r \mathrm{~cm}$, where $R_{\text {in }}=3 R_{\mathrm{S}} \approx$ 
$9 \times 10^{14} M_{9} \mathrm{~cm}$ is the inner radius of the jet, $R_{\mathrm{S}}$ is the Schwarzschild radius of the black hole, $M_{\mathrm{BH}}=10^{9} M_{9} \mathrm{M}_{\odot}$ is the mass of the black hole in the Solar masses, $\xi=0.1 \xi_{-1}$ is the scaling factor of the re-connection region assumed to be comparable to the perpendicular extend of the jet which is $\sim 0.1 \mathrm{r}$ for the opening angle of the order of $\sim 0.1 \mathrm{rad}$.

The mean free path for the disintegration of the Helium nuclei (expected to be the most abundant between the nuclei) in the radiation field of the accretion disk is $\lambda_{\mathrm{des}}^{\mathrm{He}}=\left(n_{\mathrm{ph}} \sigma_{\mathrm{des}}^{\mathrm{He}}\right)^{-1} \approx$ $9.4 \times 10^{11} r^{2} / T_{4.5}^{3} \mathrm{~cm}$, where $n_{\mathrm{ph}} \approx 5.3 \times 10^{14} T_{4.5}^{3} / r^{2} \mathrm{ph} . \mathrm{cm}^{-3}$ is the density of the diluted, by the factor $r$, black body radiation with the temperature at the inner disk radius $T_{\mathrm{D}}=3 \times 10^{4} T_{4.5} \mathrm{~K}$, $\sigma_{\mathrm{des}}^{\mathrm{He}} \approx 2 \times 10^{-27} \mathrm{~cm}^{2}$ is the cross section for the disintegration process of He nuclei at its maximum (see Appendix A in [19]). In order to provide an efficient disintegration of nuclei, $\lambda_{\text {des }}^{\mathrm{He}}$ should be shorter than the size of the re-connection region. In contrary, helium nuclei have to be accelerated to large enough energies in order to suffer efficient disintegration. The electric field strength within the re-connection region is parametrised by, $V_{\text {rec }}=\eta c B \approx 3 \times 10^{3} \eta_{-1} B_{2} r^{-\beta} \mathrm{V} \mathrm{cm}^{-1}$, where the magnetic field strength along the jet is described by $B=B_{\text {in }} r^{-\beta}$ (with $\beta=1$ for the toroidal component and $\beta=2$ for the longitudinal component), the magnetic field at the base of the jet is $B_{\text {in }}=100 B_{2} \mathrm{G}, \eta=0.1 \eta_{-1}$ is the efficiency of the re-connection process, and $c$ is the velocity of light. The maximum Lorentz factors of the nuclei can be estimated from, $E_{\mathrm{He}}^{\max }=V_{\mathrm{rec}} L_{\mathrm{rec}} Z_{\mathrm{He}} \approx 3 \times 10^{17} \eta_{-1} \xi_{-1} M_{9} B_{2} Z_{\mathrm{He}} r^{1-\beta} \mathrm{eV}$. where $Z_{\mathrm{He}}=2 e$ is the charge of He nuclei and $e$ is the elementary charge.

The He nuclei are efficiently disintegrated when the soft radiation from the accretion disk reaches energies above $E_{\mathrm{min}}^{\mathrm{He}} \sim 20 \mathrm{MeV}$ in their reference frame (e.g. see Appendix A in [?]). This condition is met for the energies of the nuclei of the order of, $E_{\mathrm{He}}^{\min }=\frac{m_{\mathrm{p}} c^{2} A_{\mathrm{He}} E_{\min }^{\mathrm{He}}}{3 k_{\mathrm{B}} T_{\mathrm{D}}} \approx \frac{2.4 \times 10^{15} A_{\mathrm{He}}}{T_{4.5}} \mathrm{eV}$, where $A_{\mathrm{He}}=4$ is the mass number of He nuclei, $m_{\mathrm{p}} c^{2}$ is the proton rest energy, and $k_{\mathrm{B}}$ is the Boltzmann constant. Note that neutrons from the disintegration of the nuclei are expected to have Lorentz factors similar to those of the parent nuclei. We assume that a part of neutrons, liberated from nuclei, impinges onto the accretion disk. Neutrons with the Lorentz factors above $\gamma_{\mathrm{n}}>E_{\mathrm{He}}^{\min } /\left(A_{\mathrm{He}} m_{\mathrm{p}}\right)$, can travel characteristic distances of the order of $X_{\mathrm{n}}>c \tau_{\mathrm{n}} \gamma_{\mathrm{n}}>8 \times 10^{18} \mathrm{~cm}$. We conclude that in most cases the neutrons extracted from the nuclei, moving towards the accretion disk, can reach the disk surface before decaying.

Essential role in the collimation of a jet plays the surrounding matter and/or the wind from the accretion disk. Therefore, jets are expected to have regions (boundaries) filled with plasma moving with various velocities. The jet likely have a faster inner section, powered by the black hole or the inner disk, and a slower outer section anchored in the more distant parts of the disk. The border between these two parts of the jet has been proposed to provide good conditions for the acceleration of particles. In fact, the acceleration of particles at the boundary between relativistic jet and its cocoon was discussed [20, 21, 22, 23, Nuclei accelerated in such mechanism should spend significant time within the cocoon of the jet in which their distribution is close to isotropic. Therefore, they can preferably interact with the nearby accretion disk radiation. As a result, the nuclei can lose nucleons in the photo-disintegration process. Neutrons can propagate towards the accretion disk and interact with the disk matter.

The simulation of the particle acceleration process [20] shows that in favorable conditions considered acceleration process can be very rapid. The acceleration length in the observer's frame, 
due to the particle advection along the jet flow, can be $L_{\mathrm{acc}} \sim \alpha_{\mathrm{o}} r_{\mathrm{L}}$, where $r_{\mathrm{L}} \approx 6 \times 10^{6} \gamma_{\mathrm{He}} B_{\mathrm{G}}^{-1} \mathrm{~cm}$ is the Larmor radius of the nuclei and the parameter $\alpha_{\mathrm{o}}$ can be as small as $\sim 10$. We are interested in situations in which the accelerated nuclei are efficiently disintegrated. Then, the acceleration length of nuclei, $L_{\mathrm{acc}} \sim 6 \times 10^{6} \alpha_{\mathrm{o}} \gamma_{\mathrm{He}} B_{\mathrm{G}}^{-1} \sim 1.8 \times 10^{10} \alpha_{\mathrm{o}} T_{4.5} r^{\beta} B_{2}^{-1} \mathrm{~cm}$, should be at least equal (or shorter) than the mean free path for their disintegration, provided that on this distance scale the nuclei reach energy above the threshold for disintegration. The condition, $L_{\mathrm{acc}} \leq \lambda_{\mathrm{des}}^{\mathrm{He}}$, is fulfilled for the distance from the base of the jet, $r \geq 0.02 \alpha_{0} T_{4.5}^{4} B_{2}^{-1}$, for the case of the dominant toroidal structure of the magnetic field in the jet, i.e. $\beta=1$ and $\alpha_{0} \sim 10$.

We estimate the maximum Lorentz factors of nuclei, accelerated at a specific distance, L, from the base of the jet, in the jet boundary layer by comparing their characteristic acceleration length, $L_{\mathrm{acc}}$, with the distance scale along the jet, $L \approx 10^{15} M_{9} r \mathrm{~cm}, \gamma_{\mathrm{He}}^{\max } \approx 1.5 \times 10^{10} M_{9} B_{2} r^{1-\beta} / \alpha_{\mathrm{o}}$. Assuming $\alpha_{\mathrm{o}} \sim 10$, this maximum Lorentz factor is $\gamma_{\mathrm{He}}^{\max } \approx 1.5 \times 10^{9} M_{9} B_{2}$ for $\beta=1$ and $\gamma_{\mathrm{He}}^{\max } \approx$ $1.5 \times 10^{9} M_{9} B_{2} / r$ for $\beta=2$. The mechanism of particle acceleration at the jet boundary described above is expected to produce relativistic particles with a flat power law spectrum [20, 21, 22, 23].

\section{Neutrons in the accretion disk}

A part of neutrons is directed towards the accretion disk. These neutrons have large enough Lorentz factors to reach the accretion disk before decaying. Neutrons have the power law spectrum consistent with the spectrum of the accelerated nuclei. We assume that the accretion disks around SMBHs in radio loud active galaxies are well described by the Shakura-Sunyaev disk model [24]. The surface mass density in the inner part of such a geometrically thin type disk is, $\Sigma(r)=4.6 \alpha^{-1} \dot{m}^{-1} r^{3 / 2}\left(1-r^{-1 / 2}\right)^{-1} \mathrm{~g} \mathrm{~cm}^{-2}$, (see Eq. 2.9 in [24]), where $r$ is the distance from the black hole expressed in units of the inner radius of the accretion disk, $r_{\text {in }}=3 r_{\mathrm{S}}=6 G M_{\mathrm{BH}} / c^{2}$, $\dot{m}$ is the accretion rate in units of the Eddington accretion rate, $\alpha$ is the viscosity coefficient, $G$ is the gravitational constant. The above formula is valid for distances, $r<150(\alpha m)^{2 / 21} \dot{m}^{16 / 21}$, where $m=M_{\mathrm{BH}} / M_{\odot}$. The density of matter in the disk is low since the thickness, $z$, of the disk around the super-massive black hole is quite large (see Eq. 2.8 in [24]), $z(r) \approx 3.2 \times 10^{6} \dot{m m}\left(1-r^{-1 / 2}\right) \mathrm{cm}$. For the parameters, $\dot{m}=0.1$ and $m=10^{9}$, the half thickness of the disk is $z \approx 9.4 \times 10^{13} \mathrm{~cm}$. Density of the matter in the disk is a few orders of magnitudes lower than the density of the Earth's atmosphere. Therefore, pions and muons with considered energies decay onto neutrinos before interacting with the matter.

We calculate the all flavor neutrino spectra produced in the above discussed scenario for the SMBHs with different masses. As an example, we assume that nuclei are accelerated with the power law spectrum and an exponential cut-off. Neutrons are extracted from these nuclei with a similar spectrum. It is of the form, $d N_{\mathrm{n}} / d E_{\mathrm{n}} \propto E_{\mathrm{n}}^{-\delta} \exp \left(-E_{\mathrm{n}} / E_{\mathrm{n}}^{\max }\right)$. The power taken by neutrons can be obtained by relating it to the accretion luminosity of the SMBH. This accretion luminosity can be again related to the Eddington luminosity of the SMBH, assuming the cosmological redshift averaged accretion efficiency, $L_{\mathrm{acc}}=\chi L_{\mathrm{Edd}}=1.3 \times 10^{46} \chi_{-1} M_{9} \mathrm{erg} \mathrm{s}^{-1}$, where $L_{\text {Edd }}$ is the Eddington luminosity, and the efficiency of the accretion process is $\chi=0.1 \chi_{-1}$. In the case of the radio-loud AGN this value has been estimated to be in the range $\sim(0.01-0.1)[25]$. It is assumed that approximately a half of the energy released by accretion is irradiated from the accretion disk surface, $L_{\mathrm{D}}=0.5 L_{\mathrm{acc}}$, and a half of the accretion energy is transferred to the jet, $L_{\mathrm{j}}=0.5 L_{\mathrm{acc}}$. 

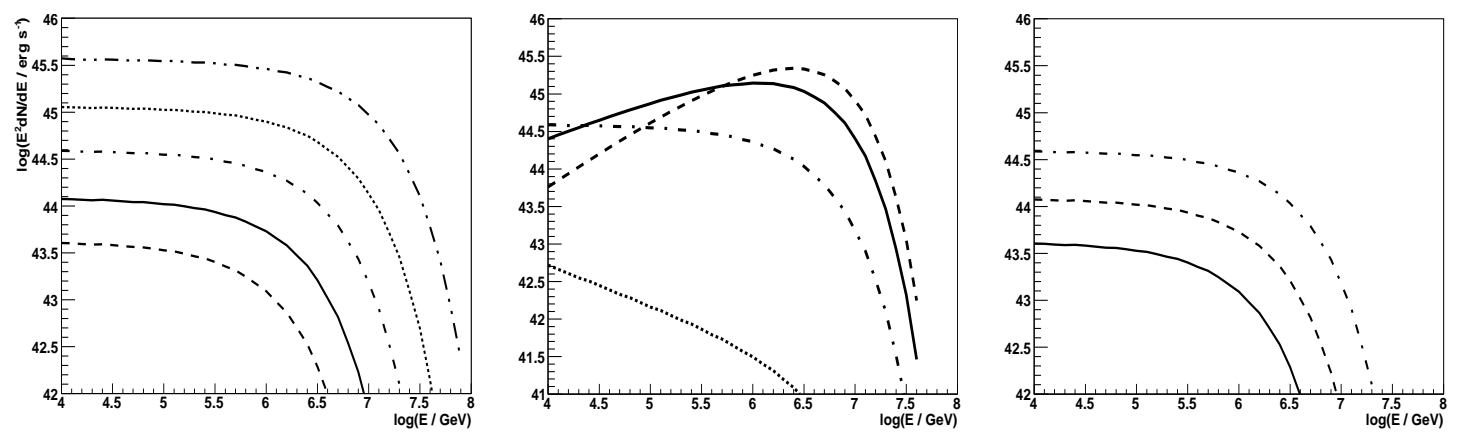

Figure 1: Neutrino (all flavor) spectra produced by neutrons in collisions with the matter of the optically thick (and geometrically thin) Shakura-Sunyaev type accretion disk for different masses of the supermassive black hole $M_{\mathrm{BH}}=10^{10} \mathrm{M}_{\odot}$ (dot-dot-dashed), $3 \times 10^{9} \mathrm{M}_{\odot}$ (dotted), $10^{9} \mathrm{M}_{\odot}$ (dot-dashed), $3 \times 10^{8} \mathrm{M}_{\odot}($ solid) and $10^{8} \mathrm{M}_{\odot}$ (dashed) (on the left). The neutrons have a power law spectrum with spectral index equal to $\delta=2$ and an exponential cut-off. The neutron spectrum is calculated assuming that the jet takes a half of the gravitational energy of the accreting matter. The black hole accretes the matter with the efficiency equal to $\chi=0.1$ of the Eddington accretion rate. $10 \%$ of the jet power is transferred to relativistic He nuclei. The neutrino spectra for different spectral indexes of neutrons, equal to $\delta=1$ (dashed curve), 1.5 (solid), 2.0 (dot-dashed), and 2.5 (dotted), are shown in the middle figure. The SMBH mass is fixed on $10^{9} \mathrm{M}_{\odot}$ and the other parameters are as defined above. The spectra for different efficiency of the accretion process, $\chi=0.1$ (dot-dashed curve), 0.03 (dashed), and 0.01 (solid), are shown on the right figure.

Assuming the Shakura-Sunyaev disk model, we estimate the characteristic temperature of the radiation emitted from the inner disk on, $T_{\mathrm{D}}=\left(\frac{L_{\mathrm{D}}}{4 \pi r_{\mathrm{in}}^{2} \sigma_{\mathrm{SB}}}\right)^{1 / 4} \approx 5.5 \times 10^{4} \frac{\chi_{-1}^{1 / 4}}{M_{9}^{1 / 2}} \mathrm{~K}$. In order to estimate the characteristic maximum energies of neutrons, $E_{\mathrm{n}}^{\max }$, as a function of the SMBH mass, we set the upper limit on the magnetic field strength at the base of the jet by assuming that the jet power is mainly curried out in the form of the Poynting flux, $L_{\mathrm{j}} \approx \pi r_{\mathrm{in}}^{2} c\left(B_{\mathrm{in}}^{2} / 8 \pi\right)^{2}$. This relation allows to estimate the magnetic field on, $B_{\text {in }} \approx 1.3 \times 10^{3}\left(\chi_{-1} / M_{9}\right)^{1 / 2} \mathrm{G}$. Introducing this value of the magnetic field strength to the formula for the maximum energies to which nuclei can be accelerated in the jet and applying the assumed scaling values for other parameters, we estimate the characteristic maximum energies of neutrons on, $E_{\mathrm{n}}^{\max }=2 \times 10^{9}\left(\chi_{-1} M_{9}\right)^{1 / 2} \mathrm{GeV}$. We fixed the parameter describing the distribution of the magnetic field in the jet on $\beta=1$. Then, the maximum energies of the nuclei are independent on the distance from the SMBH. A part of the neutrons, extracted from the nuclei, move towards the accretion disk and interact with the matter producing neutrinos in hadronic collisions. As an example, we show the dependence of the neutrino spectra on the SMBH mass (see Fig. 1 on the left), on the spectral index of the spectrum of nuclei (see Fig. 1 in the middle), and different efficiency of the accretion process (see Fig. 1 on the right). Note that the spectra of particles with indexes as low as $\delta=1$ are expected in the case of the re-connection process [?]. The spectra shown in the first two figures on Fig. 1, are calculated for the SMBH accreting the matter at the rate of $\chi=10 \%$ of the Eddington accretion rate and assuming that the nuclei take $10 \%$ of the jet power. 


\section{Extragalactic neutrino background}

In order to determine the contribution of the neutrinos, produced in the accretion disks around SMBHs, to the Extragalactic Neutrino Background ENB, we integrate the neutrino spectra over the luminosity function of the spheroids around SMBHs in active galaxies. This luminosity function is related to the masses of SMBHs. We integrate this formula over the part of the Universe up to the redshift $z_{\max }=2$. The diffuse neutrino flux is then given by,

$$
\Phi_{v}=\frac{c}{4 \pi H_{0}} \int_{0}^{z_{\max }} \frac{d z}{\left[(1+z)^{3} \Omega_{\mathrm{m}}+\Omega_{\Lambda}\right]^{1 / 2}} \int d L \frac{d N(L, z)}{d L d V} \frac{d N_{v}\left(E_{v}(1+z)\right)}{d E_{v} d t}
$$

where $d N(L, z) / d L d z=(d N(L, z) / d L d V)(d V / d z)$ is the spheroid luminosity, $L$, function, $z$ is the redshift, and $V$ is the volume. The spheroid luminosity is related to the SMBH mass through the formula,

$$
\log \frac{M_{\mathrm{BH}}}{M_{\odot}}=1.13 \log \frac{L}{L_{\odot}}-4.11-0.316 z+1.4 \log (1+z),
$$

taken from [26]. The luminosity function is expressed as a Schechter function [27],

$$
\frac{d N(L, z)}{d L d V}=\frac{\Phi_{0}(z)}{L_{\star}}\left(\frac{L}{L_{\star}}\right)^{-1.07} \exp \left(-\frac{L}{L_{\star}}\right),
$$

where the function, $\Phi_{0}(z)=3.5 \times 10^{-3} \exp \left[-(z / 1.7)^{1.47}\right] \mathrm{Mpc}^{-3}$ is given by Eq. 12 in [26] and $L_{\star}(z)=1.4 \times 10^{11} 10^{0.4\left[(z / 1.78)^{0.47}\right]} \mathrm{L}_{\odot}$ is given by Eq. (A6) in [26]. In our calculations, we apply $\Omega_{\mathrm{m}}=0.3, \Omega_{\Lambda}=0.7$, and $H_{0}=70 \mathrm{~km} \mathrm{~s}^{-1} \mathrm{Mpc}^{-1}$. For the specific luminosity of spheroid galaxy, we derive the mass of the SMBH and determine the accretion luminosity. The extragalactic neutrino background is calculated after integrating over the population of the SMBHs within the active galaxies. The results are compared on Fig. 2, with the all-flavor ENB reported by the IceCube [28], assuming different spectral indexes of the accelerated nuclei (on the left) and different values of the accretion efficiency onto the SMBHs (Fig. 2 on the right). The best description of the ENB is obtained for the spectral index equal to $\delta=2.2$ and rather large accretion efficiency $\chi=0.1$. The level of calculated ENB is consistent with the observations provided that the normalization factor of the spectrum of nuclei is equal to $A_{N} \sim 2 \times 10^{-6}$. The factor $A_{N}$ is the product of a few coefficients, i.e. the accretion power of the SMBH (in units of the Eddington luminosity) expressed by $\chi$, a factor describing the part of energy of nuclei taken by neutrons (in the range $\sim 8^{-1}$ to $\sim 2^{-1}$ ), the part of the accretion power transferred to the jet (assumed $2^{-1}$ ), the efficiency of acceleration of nuclei (usually assumed 10\%), the parameter describing a part of all SMBHs which are active within galaxies, and the parameter describing a part of neutrons which reach the accretion disk. The last two factors are the most uncertain. For the values of the other coefficients mentioned above, the product of these two last parameters should be of the order of $(0.8-3.2) \times 10^{-3}$. Therefore, the model can explain the observations of the ENB provided that e.g. $(1.6-6.4) \%$ of the neutrons reach the accretion disk surface and 5\% of SMBHs are in the active phase.

We have also investigated the effect of much lower accretion efficiency of matter onto the SMBHs by showing the neutrino spectra for $\chi=0.03$ and different spectral indexes of the neutrons (Fig. 2 on the right). It is clear that the lower accretion efficiency might be also consistent with 

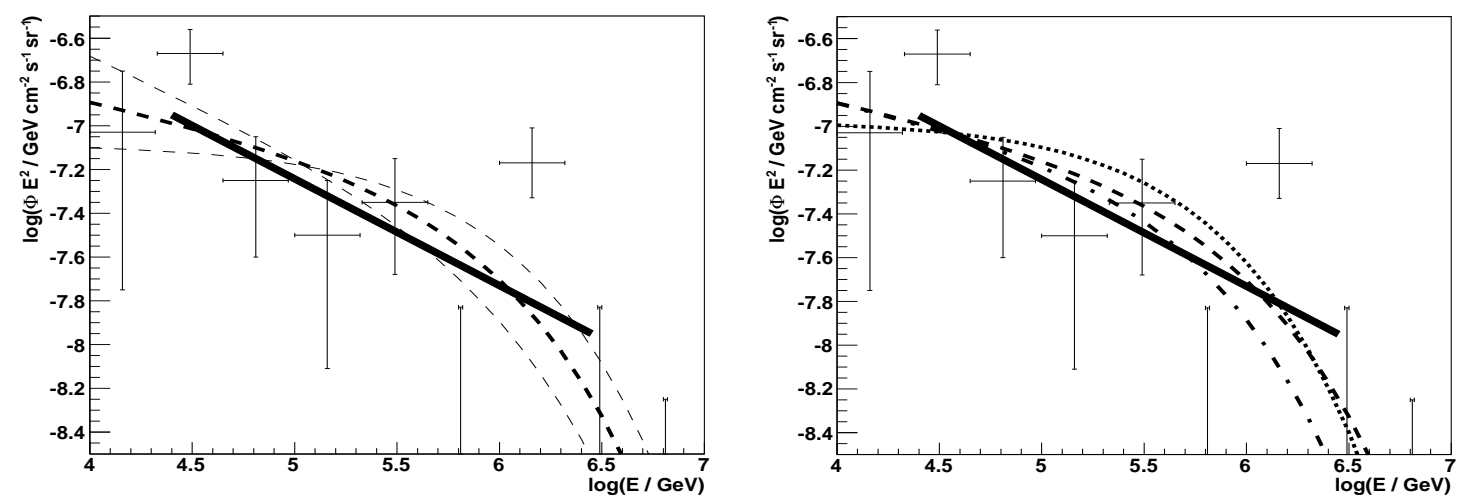

Figure 2: Extragalactic (all flavor) Neutrino Background (ENB) produced by neutrons in collisions with the matter of the optically thick accretion disks around black holes in the Universe up to the redshift of $z_{\max }=2$. The ENB, calculated for the power law spectrum of neutrons with the exponential cut-off at $E_{\mathrm{n}}^{\max }$ and spectral index equal to $\delta=2.2$ (thick dashed curve), and 2. and 2.4 (thin dashed curves), is shown on the left figure. The normalization factor of the spectra is equal to $A_{N} \approx 2 \times 10^{-6}, 3 \times 10^{-7}$, and $2 \times 10^{-5}$, respectively for the spectral indexes mentioned above. The spectrum of neutrons extends between $\gamma_{n}^{\min }$ and $\gamma_{\mathrm{n}}^{\max }$. The efficiency of the accretion process onto the SMBHs is fixed on $\chi=0.1$. The population of the super-massive black holes in the Universe is modeled as described in Sect. 4. The dependence of the ENB on the efficiency of the accretion process, for $\chi=0.1$ and $\delta=2.2$ (dashed curve), 0.03 and $\delta=2$ (dotted, $A_{\mathrm{N}} \approx 10^{-6}$ ), 0.03 and $\delta=2.2$ (dot-dashed, $A_{\mathrm{N}} \approx 6 \times 10^{-6}$ ), is shown on the right figure. The reported spectrum of (all flavor) neutrino background is shown by the solid error bars and its power law model by the thick solid line [28].

the observations provided that the spectral index of accelerated nuclei is clearly flatter than the previously considered value 2.2 (taking into account large error bars of the neutrino spectral points).

The neutrinos produced in terms of such a model are expected to be only mildly beamed in the direction perpendicular to the accretion disk. Therefore, different types of observed active galaxies, i.e. blazars, radio galaxies, or even Seyfert galaxies with evidences of jets, could contribute to the ENB. Due to this feature of unbeamed neutrino emission, only the closest AGNs might be expected to produce observable neutrino event rates in the IceCube telescope. In order to check this, we calculate expected neutrino event rates from the nearby active galaxy, M 87, under the hypothesis that it is typical contributor to the ENB, i.e. assuming for it the average normalization factor $A_{\mathrm{N}}=2 \times 10^{-6}$ derived from the modeling of the ENB. For the active galaxy M87, which is at the distance of $16.4 \mathrm{Mpc}[29]$ and harbors a SMBH with the mass $\sim 5 \times 10^{9} \mathrm{M}_{\odot}$ [30], the expected neutrino event rate in the IceCube telescope is estimated on $\sim 0.7 v \mathrm{yr}^{-1}$. We used the effective area of the IceCube neutrino detector reported in [31]. In these calculations we assumed the spectral index of nuclei equal to 2, i.e. marginally consistent with the modeling of the ENB (see Fig. 2). Note however, that M 87 is under-luminous active galaxy with the estimated accretion rate $\dot{m} \approx 1.6 \times 10^{-3}[32]$. The accretion process in M87 may not be correctly described by the considered in this paper Shakura-Sunyaev disk model. Therefore, this neutrino event rate should be considered as the upper limit. We conclude that the perspectives for the identification of the neutrino events with the specific active galaxies are not very promising in terms of the considered model. 


\section{Acknowledgements}

This work is supported by the grant through the Polish NCN No. 2014/15/B/ST9/04043.

\section{References}

[1] M.G. Aartsen et al. Science 342 (2013) 1

[2] M.G. Aartsen et al. PRL 113 (2014) 101101

[3] K. Murase in "Neutrino Astronomy - Current status, future prospects", Eds. T. Gaisser \& A. Karle (World Scientific) [arXiv:1511.01590]

[4] J. Becker Physics Reports 458 (2008) 173

[5] L. Nellen, K. Mannheim, P.L. Biermann PRD 47 (1993) 5270

[6] W. Bednarek Astrophys. J. 833 (2016) 279

[7] R.D. Blandford, R.L. Znajek MNRAS 179 (1977) 433

[8] R.D. Blandford MNRAS 176 (1976) 465

[9] R.V.E. Lovelace Nature 262 (1976) 649

[10] M. Peimberet, V. Luridiana, A. Peimbert ApJ 666 (2007) 636

[11] M.M. Romanova, R.V.E. Lovelace $A \& A 262$ (1992) 26

[12] H. Lesch, G.T. Birk ApJ 499 (1998) 167

[13] D.E. Larrabee, R.V.E. Lovelace, M.M. Romanova ApJ 586 (2003) 72

[14] M. Lyutikov NewAR 47 (2003) 513

[15] C.H. Jaroschek, H. Lesch, R.A. Treumann ApJ 605 (2004) L9

[16] D. Giannios, D.A. Uzdensky, M.C. Begelman MNRAS 395 (2009) 29

[17] L. Sironi, A. Spitkovsky ApJ 783 (2014) L21

[18] W. Bednarek, J.G. Kirk, A. Mastichiadis A\&A 307 (1995) L17

[19] R.H. Cyburt et al. PRD 67 (2003) 103521

[20] M. Ostrowski $A \& A 238$ (1990) 435

[21] M. Ostrowski A\&A 335 (1998) 134

[22] M. Ostrowski MNRAS 312 (2000) 579

[23] F.M. Rieger, P. Duffy ApJ 652 (2006) 1044

[24] Shakura, N.I., Sunyaev, R.A. 1973 A\&A 24, 337

[25] X.-B. Wu, F.K. Liu ApJ 614 (2004) 91

[26] Y.-R.Li, L.C. Ho, J.-M. Wang ApJ 742 (2011) 33

[27] P. Schechter ApJ 203 (1976) 297

[28] M.G. Aartsen et al. ApJ 809 (2015) 98

[29] S. Bird et al. A\&A 524 (2010) 71

[30] J.L. Walsh et al. ApJ 770 (2013) 86

[31] M.G. Aartsen et al. APh 66 (2015) 39

[32] T. di Matteo et al. ApJ 582 (2003) 133 